\title{
Interlayer dielectric function of a type-II van der Waals semiconductor: the $\mathrm{HfS}_{2} / \mathrm{PtS}_{2}$ hetero-bilayer
}

\author{
Stefana Anais Colibaba,,${ }^{1 *}$ Sabine Körbel, ${ }^{1}$ Carlo Motta, ${ }^{1}$ Fedwa El-Mellouhi, ${ }^{2}$ and Stefano Sanvito ${ }^{1, \dagger}$ \\ ${ }^{1}$ School of Physics, AMBER and CRANN Institute, Trinity College, Dublin 2, Ireland \\ ${ }^{2}$ Qatar Environment and Energy Research Institute, PO Box 5825, Doha, Qatar
}

(Dated: November 20, 2019)

\begin{abstract}
Heterogeneous stacks of two-dimensional transition-metal dichalcogenides can be arranged so as to have a type-II band alignment, where the valence band maximum and the conduction band minimum are located on different layers. These structures can host long-living inter-layer excitons with inhibited charge recombination and enhanced charge-carrier separation. Inter-layer excitons appear as photoluminescence peaks below the band gap, but not in absorption experiments, indicating that they may form after and not during absorption. In order to quantify the inter-layer component of the absorption spectra of such heterostructures, we perform first-principles calculations of the layerdecomposed dielectric function of the $\mathrm{HfS}_{2} / \mathrm{PtS}_{2}$ hetero-bilayer. This has a type-II band alignment and a relatively small inter-layer distance, which should facilitate the formation of inter-layer excitons. We find that the inter-layer component is always only a small fraction of the total dielectric function, owing to the large spatial separation between the electron and the hole. However, the inter-layer contribution is greatly enhanced upon reducing the interlayer distance. Compression of the layers produces a split-off band at the top of the valence bands. This remains localised on $\mathrm{PtS}_{2}$ so that the heterostructure preserves the type-II character. At the same time the type-II bandgap is reduced, moving the inter-layer absorption peak to a lower energy and to a position well separated from the rest of the absorption spectrum.
\end{abstract}

\section{INTRODUCTION}

Two-dimensional transitional metal dichalcogenides (2D TMDs) are a very suitable materials class for photovoltaic (PV) applications, because of their welldeveloped production techniques and generally attractive electronic properties $[1,2]$. Many of the known 2D TMDs have a semiconductor bandstructure and possess similar bandgaps in the visible to near infrared range, high carrier mobility, strong photoluminescence and exciton binding energies that can be tuned with the number of layers that are stacked on top of each other. These are all features that make them strong candidates for various optoelectronic devices such as solar cells, photo-detectors and light-emitting diodes [3]. In general, 2D materials are layered compounds characterised by a crystalline planar structure held together by strong in-plane covalent bonds and weak out-of-plane van der Waals (vdW) forces [4]. This peculiar configuration allows them to be exfoliated into thin planes or monolayers (MLs) by either mechanical or chemical means, and to be re-assembled on top of each other to build a multitude of different vdW heterostructures. Such heterostructures can be designed layer by layer without being constrained by the lattice mismatch $[3,5,6]$, a manufacturing flexibility that enables the practical realization of novel properties otherwise difficult to obtain.

For instance one can engineer heterogeneous bilayers (HBLs) having the so-called type-II band alignment,

\footnotetext{
* colibaba@tcd.ie

† sanvitos@tcd.ie
}

where the conduction band minimum (CBM) resides on one layer type, while the valence band maximum (VBM) is on the other (see Fig. 1). In this case spatial separation of the electron-hole pairs (excitons) is possible, such that the ground state of the exciton (the thermalised exciton) ends up having the charge carriers of different polarity located on the two different layer types. Such inter-layer excitons may form either already during the light absorption, or afterwards as the product of scattering. If they form during absorption, some inter-layer transitions might appear as additional features in the absorption spectra, overall increasing the absorption efficiency. In type-II HBLs, inter-layer transitions could occur at an energy below the absorption or emission edges of the individual layers (intra-layer transitions). This is the most favourable situation for detection as no other transitions are available in that spectral range. Inter-layer excitons recombine more slowly compared to intra-layer ones, a feature that can be detected experimentally. Such longliving inter-layer excitons have indeed been previously identified in TMDs HBLs in photoluminescence experiments [7-10].

The formation process of inter-layer excitons in vdW materials is still debated. Some studies point to the existence of inter-layer excitations in the absorption spectra. For instance in Ref. [1] Bernardi et al. state that the absorption onset of the $\mathrm{MoS}_{2} / \mathrm{WS}_{2}$ bilayer, as calculated with highly-accurate many-body perturbation theory, is shifted to a lower energy as compared to that of monolayers. This feature is taken as a signature of an interlayer charge-transfer excitation. In contrast, other works report the absence of inter-layer effects in the HBLs absorption spectra $[9,11,12]$.

Andersen et al. [13] proposed a model for comput- 


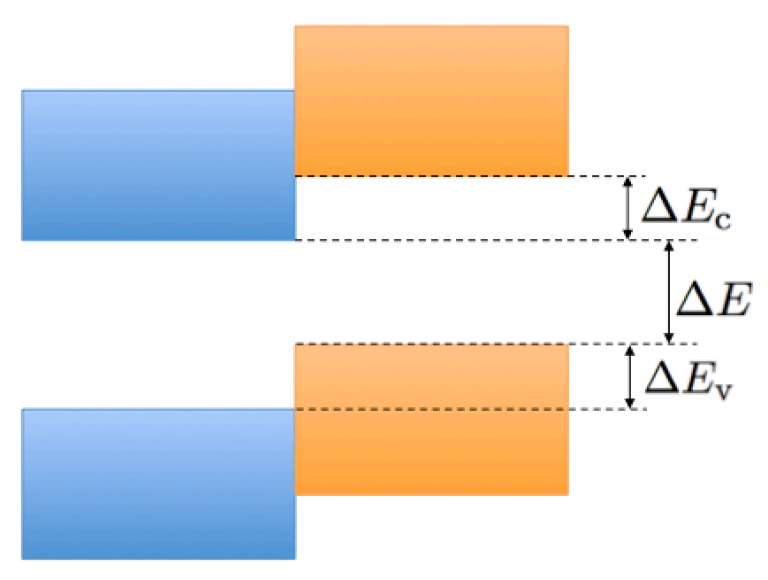

FIG. 1. (Colour online) Type-II band alignment. The differently coloured blocks represent the valence and conduction bands of the two monolayers making up the bilayer. The bilayer bandgap, $\Delta E$, is between the valence band maximum of one layer type and the conduction band minimum of the other. The conduction and valence band offsets between the individual layers are denoted as $\Delta E_{\mathrm{c}}$ and $\Delta E_{\mathrm{v}}$, respectively.

ing the dielectric function of multi-layer systems based on ab initio calculations for monolayers combined with a classical electrostatic model for dielectric screening. Interlayer hybridization is not included in the model, which nonetheless yields a remarkably good agreement with full ab initio calculations of the dielectric function. This confirms that interlayer coupling is largely a screening effect and that electronic inter-layer transitions are only weak. Komsa et al. performed first-principles calculations of the dielectric function of $\mathrm{MoS}_{2} / \mathrm{WS}_{2}$ heterostructures with many-body perturbation theory in order to accurately capture excitonic effects. They found that electronic interlayer excitations are very weak, and that the absorption spectrum of the HBL resembles a superposition of those of the constituent MLs [14].

In general, screening effects are hard to distinguish from inter-layer excitons in absorption spectra of type II heterostructures. This is because they can both lead to a bandgap reduction and hence to a red-shift in the absorption spectra. In this work, in order to separate screening effects from interlayer transitions, we decompose the dielectric function, as calculated with ab initio density functional theory (DFT), into inter-layer and intra-layer components. Our heterostructure of choice is $\mathrm{HfS}_{2} / \mathrm{PtS}_{2}$, a decision motivated by two main reasons. On the one hand, $G W$ calculations for the constituent monolayers exist [15] and suggest that the HBL is indeed of type II. On the other hand $\mathrm{HfS}_{2}$ and $\mathrm{PtS}_{2}$ have an almost identical in-plane lattice parameter so that a commensurate bi-layer unit cell can be constructed without the need for large supercells.

The remainder of the paper is organised as follows. In section II we describe the methods and computational details of our calculations. Then, in section III we present and discuss our results for the bandstructures and the layer-resolved dielectric function of the $\mathrm{HfS}_{2} / \mathrm{PtS}_{2} \mathrm{HBL}$, of the constituent MLs and of the homogeneous $\mathrm{HfS}_{2}$ and $\mathrm{PtS}_{2}$ bilayers. Here we analyse the effects of the layer spacing and of the light polarization direction on the strength of the inter-layer transitions. The paper closes with a summary and an outlook.

\section{METHODS AND COMPUTATIONAL DETAILS}

All the calculations have been performed with DFT $[16,17]$. We have chosen the specific numerical DFT implementation contained in the FHI-AIMS allelectron package $[18,19]$ that adopts a numerically tabulated atom-centered orbitals basis set [20]. In order to ensure sufficient accuracy we have used the "tight" basis set constructed using the first tier numerical orbitals for the metal atoms and a modified version of the first tier for the sulphur atoms. Geometry relaxations have been performed with an all-electron potential and the generalised gradient approximation (GGA) of the exchange and correlation energy, as parametrised by Purdew, Burke and Ernzerhof (PBE) [21]. An energy convergence threshold of $10^{-6} \mathrm{eV}$ for the Kohn-Sham self-consistency cycle. The van der Waals interaction between layers has been taken into account by using the Tkatchenko-Scheffler van der Waals correction scheme [22]. A force convergence threshold of $10^{-5} \mathrm{eV} / \AA$ and a $10 \times 10 \times 1 k$-mesh have been used for the structural optimization of the $\mathrm{HfS}_{2}$ and $\mathrm{PtS}_{2}$ MLs.

Ground-state bandstructure calculations have been carried out with PBE and also with the hybrid HeydScuseria-Ernzerhof (HSE06) [23] exchange-correlation functional. Since the absorption spectrum is highly sensitive to the size of the direct bandgap, we have also employed HSE06 for the calculation of the dielectric function. In fact, HSE06 typically corrects for the bandgap underestimation produced by semilocal functionals such as PBE. We have obtained the layer-projected bandstructure of the $\mathrm{HfS}_{2} / \mathrm{PtS}_{2}$ HBL with VASP, the Vienna $\mathrm{Ab}$ initio Simulation Package [24]. VASP uses pseudopotentials and it is based on the projector augmented wave method. In this case the number of valence electrons for each distinct species is as follows: 12 for hafnium, 16 for platinum and 6 for sulphur. We have employed a $10 \times 10 \times 1$ Monkhorst-pack mesh and a plane-wave cutoff energy of $400 \mathrm{eV}$.

Since the in-plane lattice parameters of the relaxed $\mathrm{HfS}_{2}$ and $\mathrm{PtS}_{2}$ monolayer are not identical $\left(a_{0}^{\mathrm{HfS}_{2}}=3.64\right.$ $\AA$ and $a_{0}^{\mathrm{PtS}_{2}}=3.57 \AA$ ), constructing the primitive cell for the $\mathrm{HfS}_{2} / \mathrm{PtS}_{2} \mathrm{HBL}$ imposes a small strain on the two layers. We have computed the HBL in-plane lattice parameters as follows. Firstly, the total energy of the two MLs, $\mathrm{HfS}_{2}$ and $\mathrm{PtS}_{2}$, has been calculated as a function of the in-plane strain, and fitted to a quadratic equation. 


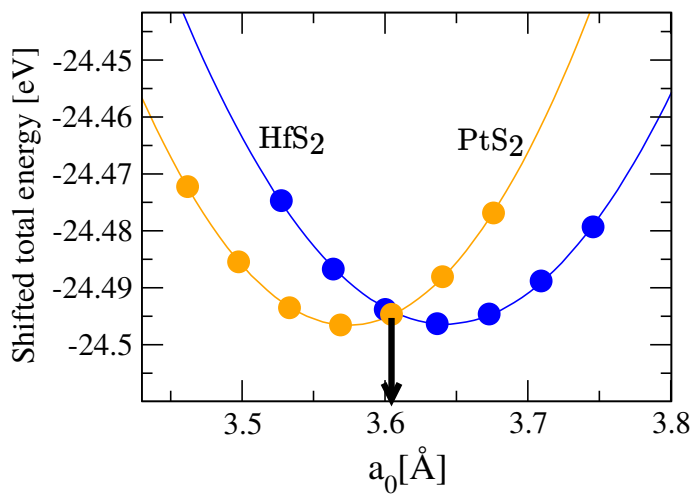

FIG. 2. (Colour online) Total energy as a function of compressive/tensile strain for monolayers of $\mathrm{HfS}_{2}$ (blue) and $\mathrm{PtS}_{2}$

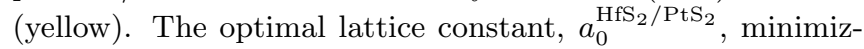
ing the total strain energy is shown by an arrow.

We have then minimised the sum of such quadratic functions in order to find the optimal in-plane lattice constant of the HBL. This is the one that minimises the total strain energy and it is found to be $a_{0}^{\mathrm{HfS}_{2} / \mathrm{PtS}_{2}=}$ $3.60 \AA$ (see arrow in Fig. 2). Throughout this work, in the case of the homogeneous $\left(\mathrm{HfS}_{2} / \mathrm{HfS}_{2}\right.$ and $\mathrm{PtS}_{2} / \mathrm{PtS}_{2}$ ) and heterogeneous $\left(\mathrm{HfS}_{2} / \mathrm{PtS}_{2}\right)$ bilayers, we have shifted the second layer relative to the first one by a distance $d$ equal to the average of the relaxed bulk $\mathrm{HfS}_{2}$ and $\mathrm{PtS}_{2}$ out-of-plane lattice parameter, namely $d=5.24 \AA$. All the fractional in-plane atomic coordinates of the MLs, homogeneous bilayers (BLs) and of the HBL are kept fixed, namely they are identical to the in-plane coordinates of the relaxed MLs relative to the $\mathrm{HfS}_{2} / \mathrm{PtS}_{2} \mathrm{HBL}$ lattice constant $a_{0}^{\mathrm{HfS}_{2} / \mathrm{PtS}_{2}}$. This is done in order to compare results for the homogeneous ML and BL systems to those for the HBL, and to single out electronic effects from those arising from structural relaxation.

For monolayers and bilayers calculations choosing an appropriate out-of-plane lattice parameter, $c$, is equivalent to choosing the size of the vacuum gap between the periodic replicas of the cell. This needs to be optimised carefully in order to obtain the correct $2 \mathrm{D}$ limit of the various quantities to calculate. In our case we have used the imaginary part of the dielectric function, $\operatorname{Im}(\underline{\underline{\varepsilon}})$, as the control quantity and set $c$ so that $\operatorname{Im}(\underline{\underline{\varepsilon}})$ converges with an error smaller than $1 \%$. The resulting $c$ was found to be $22.62 \AA$. Note that the absolute scale of the dielectric function depends on the volume of the unit cell used for the calculation [see Eq. (1)]. As such our calculated dielectric functions are not directly comparable with experiments. However, since for all the structures investigated we have maintained the same in-plane and out-ofplane lattice parameters, respectively $a_{0}^{\mathrm{HfS}_{2} / \mathrm{PtS}_{2}}$ and $c$, they can be compared directly with each other. A $k$-grid of $30 \times 30 \times 1$ points was found to converge the dielectric function reasonably well. The converged $k$-grid was established once the maximum value of the difference be- tween two spectra of different k-grids was less than $10 \%$. A Lorentzian function of width $0.1 \mathrm{eV}$ was employed to construct smooth dielectric function spectra.

Throughout this work the Cartesian components, $\varepsilon_{i j}$, of the dielectric function have been calculated by using the random-phase approximation (RPA) [25], which returns us an expression

$$
\begin{gathered}
\varepsilon_{i j}(\omega)=\delta_{i j}-\frac{4 \pi e^{2}}{V_{\mathrm{cell}} m^{2} \omega^{2}} \sum_{n, \mathbf{k}}\left(-\frac{\partial f}{\partial \epsilon}\right) p_{i, n, n, \mathbf{k}} p_{j, n, n, \mathbf{k}}^{*}+ \\
+\frac{4 \pi \hbar^{2} e^{2}}{V_{\mathrm{cell}} m^{2}} \sum_{\mathbf{k}} \sum_{\mathrm{c}, \mathrm{v}} \frac{p_{i, \mathrm{c}, \mathrm{v}, \mathbf{k}} p_{j, \mathrm{c}, \mathrm{v}, \mathbf{k}}^{*}}{\left(\epsilon_{\mathrm{c}, \mathbf{k}}-\epsilon_{\mathrm{v}, \mathbf{k}}-\omega\right)\left(\epsilon_{\mathrm{c}, \mathbf{k}}-\epsilon_{\mathrm{v}, \mathbf{k}}\right)^{2}}
\end{gathered}
$$

where $V_{\text {cell }}$ is the unit-cell volume, $e$ is the electron charge, $m$ is the electron mass, $f$ is the Fermi function, $\omega$ is the photon energy and $p_{j, n^{\prime}, n, k}$ are the momentum matrix elements between the Kohn-Sham eigenstates $\left|\psi_{n \mathbf{k}}\right\rangle$ for band $n$ and wave vector $\mathbf{k}$ with energy $\epsilon_{n, \mathbf{k}}$. These read

$$
p_{j, n^{\prime}, n, \mathbf{k}}=\left\langle\psi_{n^{\prime} \mathbf{k}}\left|-i \hbar \nabla_{j}\right| \psi_{n \mathbf{k}}\right\rangle .
$$

In Eq. (1) "c" and "v" denote the conduction and valence band, respectively.

In order to single out quantitatively the contributions to the dielectric function originating from the intra- and inter-layer transitions we have applied the following procedure. We denote with $\alpha$ and $\beta$ two distinct subsets of the basis functions placed respectively on the $\alpha$ and $\beta$ layer, namely we partition the Kohn-Sham wave functions as

$$
\left|\psi_{n \mathbf{k}}\right\rangle=\left|\psi_{n \mathbf{k}}^{\alpha}\right\rangle+\left|\psi_{n \mathbf{k}}^{\beta}\right\rangle
$$

The momentum matrix elements, Eq. (2), can be then decomposed as

$$
p_{j, n^{\prime}, n, \mathbf{k}}=p_{j, n^{\prime}, n, \mathbf{k}}^{\alpha \alpha}+p_{j, n^{\prime}, n, \mathbf{k}}^{\alpha \beta}+p_{j, n^{\prime}, n, \mathbf{k}}^{\beta \alpha}+p_{j, n^{\prime}, n, \mathbf{k}}^{\beta \beta},
$$

with $p_{j, n^{\prime}, n, \mathbf{k}}^{\alpha \beta}=\left\langle\psi_{n^{\prime} \mathbf{k}}^{\alpha}\left|-i \hbar \nabla_{j}\right| \psi_{n \mathbf{k}}^{\beta}\right\rangle$. This allows us to separate the dielectric function of Eq. (1) into three distinct components

$$
\varepsilon_{i j}(\omega)=\varepsilon_{i j}^{\text {inter }}(\omega)+\varepsilon_{i j}^{\text {intra }}(\omega)+\varepsilon_{i j}^{\operatorname{mixed}}(\omega),
$$

where the inter-layer component, $\varepsilon_{i j}^{\text {inter }}(\omega)$, is restricted to products of inter-layer matrix elements of the form $p_{i, n^{\prime}, n, \mathbf{k}}^{\alpha \beta} p_{j, n^{\prime}, n, \mathbf{k}}^{\alpha \beta *}$ and $p_{i, n^{\prime}, n, \mathbf{k}}^{\alpha \beta} p_{j, n^{\prime}, n, \mathbf{k}}^{\beta \alpha *}$, the intralayer component, $\varepsilon_{i j}^{\text {intra }}(\omega)$, contains only products between the intra-layer matrix elements of the form $p_{i, n^{\prime}, n, \mathbf{k}}^{\alpha \alpha} p_{j, n^{\prime}, n, \mathbf{k}}^{\alpha \alpha *}$ and $p_{i, n^{\prime}, n, \mathbf{k}}^{\alpha \alpha} p_{j, n^{\prime}, n, \mathbf{k}}^{\beta \beta *}$, and the "mixed" component, $\varepsilon_{i j}^{\text {mixed }}(\omega)$, is made up of all the remaining products between intra-layer and inter-layer matrix elements, $p_{i, n^{\prime}, n, \mathbf{k}}^{\alpha \alpha} p_{j, n^{\prime}, n, \mathbf{k}}^{\alpha \beta *}, p_{i, n^{\prime}, n, \mathbf{k}}^{\alpha \alpha} p_{j, n^{\prime}, n, \mathbf{k}}^{\beta \alpha *}, p_{i, n^{\prime}, n, \mathbf{k}}^{\alpha \beta} p_{j, n^{\prime}, n, \mathbf{k}}^{\alpha \alpha *}$, and $p_{i, n^{\prime}, n, \mathbf{k}}^{\beta \alpha} p_{j, n^{\prime}, n, \mathbf{k}}^{\alpha \alpha *}$

Note that the three contributions to the dielectric function introduced by our procedure do not correspond, as 
those of any other partition, to any physical observables. However, they enable us to single out transitions between conduction and valence states localised on the different layers and to study how these contributions evolve when changing the structure, or the interaction between the layers. As such, the quantities introduced in Eq. (5) have to be considered in the same spirit as the orbital populations in the Mulliken analysis [26]. The mixed component of the dielectric function may be thought as analogue to the overlap Mulliken population and it does not possess a transparent physical interpretation.

\section{RESULTS AND DISCUSSION}

\section{A. Bandstructure}

We begin by discussing the calculated bandstructures of the fully relaxed MLs and of their corresponding BLs. These are presented in Fig. 3, where we report results for $\mathrm{HfS}_{2}$ [panel (a)] and $\mathrm{PtS}_{2}$ [panel (b)], computed at both the PBE and HSE level.

$\mathrm{HfS}_{2}$ ML crystallises in the $1 \mathrm{~T}$ form (its bulk structure belongs to the trigonal system, space group $P \overline{3} m 1$, No. 164) and it is an indirect gap semiconductor with the VBM at the $\Gamma$ point and the CBM at M. The only substantial difference between the PBE and HSE results is the increased bandgap obtained with HSE, while the band curvatures (effective masses) remain essentially identical for the two functionals. The HSE-calculated bandgap is $1 \mathrm{eV}$ smaller than that computed with $G_{0} W_{0}$ starting from a local-density-approximation bandstructure $\left(G_{0} W_{0} @ L D A\right)$ as reported in Ref. [15] (see Table I for a detailed comparison of the various bandgap energies). The bandgap of $\mathrm{HfS}_{2}$ has been measured in the past with a combination of angle-resolved photoemission and inverse photoemission. Bulk $\mathrm{HfS}_{2}$ shows the significant indirect gap of $2.85 \mathrm{eV}$ between the $\Gamma$ and the $\mathrm{M}$ points [27]. Unfortunately the same measurement is not available for the ML form. In contrast a direct bandgap has been measured at $\Gamma$ for both bulk [27] and for few-layer $\mathrm{HfS}_{2}$ [28] to be around $3.6 \mathrm{eV}$ and rather independent of the number of layers. Such value, which can be extrapolated to the ML, lies in between our computed HSE result of $2.97 \mathrm{eV}$ and the $G_{0} W_{0} @ \mathrm{LDA}$ one of $3.97 \mathrm{eV}$ reported in [15]. This gives us confidence that our HSE description of the material is quantitatively sound. When forming the bilayer there is a small reduction of the bandgap (in the region of $200 / 300 \mathrm{meV}$ ). This is due partially to the dielectric screening by the other layer, and partially to the small interlayer interaction, which splits the bands. Notably, the VBM now moves slightly away from the $\Gamma$ point to locate along the $\Gamma$-M symmetry line (for both HSE and PBE).

$\mathrm{PtS}_{2} \mathrm{ML}$ also crystallises in the $1 \mathrm{~T}$ form and presents a semiconducting bandstructure with the CBM sitting along the $\Gamma-\mathrm{M}$ line, a feature common to both PBE and HSE. The VBM is also along the $\Gamma-M$ line, but the pre-

\begin{tabular}{rcccc}
\hline \hline & $\Delta E_{\mathrm{d}}^{G_{0} W_{0}}$ & $\Delta E_{\mathrm{i}}^{G_{0} W_{0}}$ & $\Delta E_{\mathrm{d}}^{\mathrm{HSE}}$ & $\Delta E_{\mathrm{i}}^{\mathrm{HSE}}$ \\
\hline $\mathrm{HfS}_{2} \mathrm{ML}$ & $3.97(\Gamma)$ & $2.98(\Gamma-\mathrm{M})$ & $2.97(\Gamma)$ & $1.98(\Gamma-\mathrm{M})$ \\
$\mathrm{HfS}_{2} \mathrm{BL}$ & - & - & $2.67(\Sigma)$ & $1.77(\Sigma-\mathrm{M})$ \\
$\mathrm{PtS}_{2} \mathrm{ML}$ & $3.14\left(\mathrm{~T}^{\prime}\right)$ & $2.95(\Sigma-\Sigma)$ & $2.61(\Sigma)$ & $2.49(\Sigma-\Sigma)$ \\
$\mathrm{PtS}_{2} \mathrm{BL}$ & - & - & $2.08(\Sigma)$ & $1.69(\Sigma-\Sigma)$ \\
$\mathrm{HfS}_{2} / \mathrm{PtS}_{2} \mathrm{HBL}$ & - & - & $2.22(\Sigma)$ & $1.41(\Sigma-\mathrm{M})$ \\
\hline \hline
\end{tabular}

TABLE I. Direct, $\Delta E_{\mathrm{d}}$, and indirect, $\Delta E_{\mathrm{i}}$, bandgaps (in eV) of $\mathrm{HfS}_{2}$ and $\mathrm{PtS}_{2}$ MLs and BLs as well as of the $\mathrm{HfS}_{2} / \mathrm{PtS}_{2}$ HBL. Results are presented for the HSE functional. We also report the results of reference [15] obtained with $G_{0} W_{0} @ L D A$ for the MLs. In that case the calculations were carried out at the relaxed geometry. In brackets we report the position in $k$-space for the direct bandgap and the position of both the VBM and CBM for the indirect ones. Note that here $\Sigma / \Sigma^{\prime}$ denotes a generic point along the $\Gamma$-M line and not the high-symmetry point at midway between $\Gamma$ and $\mathrm{M}$.

cise location is different for PBE and HSE. Notably, the $\mathrm{VBM}$ is almost degenerate, since there is another point along the $\Gamma-\mathrm{K}$ direction, which is extremely close to the valence top. Such bandstructure returns us $\mathrm{PtS}_{2} \mathrm{ML}$ as an indirect gap semiconductor, where the CBM and VBM are quite close in $k$-space. Furthermore, the direct gap is only about $150 \mathrm{meV}$ larger than the indirect one, meaning that $\mathrm{PtS}_{2} \mathrm{ML}$ is almost a direct bandgap semiconductor. The same situation is found also in $G_{0} W_{0} @ L D A$ calculations [15], and now the HSE gap (either direct or indirect) is only about $0.5 \mathrm{eV}$ smaller than that from $G_{0} W_{0} @$ LDA. We are not aware of any photoemission and inverse-photoemission experiment for $\mathrm{PtS}_{2}$, so an experimental determination of the quasi-particle gap is not available. However, the optical absorption edge of ML $\mathrm{PtS}_{2}$ has been measured [29] in the region of $1.6 \mathrm{eV}$ and found to be very sensitive to the number of layers. This is broadly consistent with our data, which returns a quasiparticle gap larger than such value, but does not provide the opportunity of establishing a full quantitative comparison.

When $\mathrm{PtS}_{2}$ is taken in its $\mathrm{BL}$ form a few significant differences appear with respect to the ML case. The most striking one is a drastic reduction of the bandgap (by more than $0.5 \mathrm{eV}$ ) arising from substantial bandsplitting. This is consistent with the strong absorption edge reduction as a function of the number of layers found in experiments [29], and points to an interlayer interaction much stronger than in the $\mathrm{HfS}_{2}$ case. Such interaction produces large distortions of the valence band, which now presents two clear quasi-degenerate VBM near $\Gamma$ along the $\Gamma-\mathrm{M}$ and $\Gamma-\mathrm{K}$ directions. In fact a closer inspection reveals that the VBM is formed by a set of $k$ points arranged arranged along a hexagon at the edge of the 2D Brillouin zone, a fact already observed before [30].

Next we move to the bandstructure of the $\mathrm{HfS}_{2} / \mathrm{PtS}_{2}$ HBL, which is presented in Fig. 4. Since the electronic interaction across the van der Waals gap is typically weak, one expects the wave-functions to be rather localised on 
a)

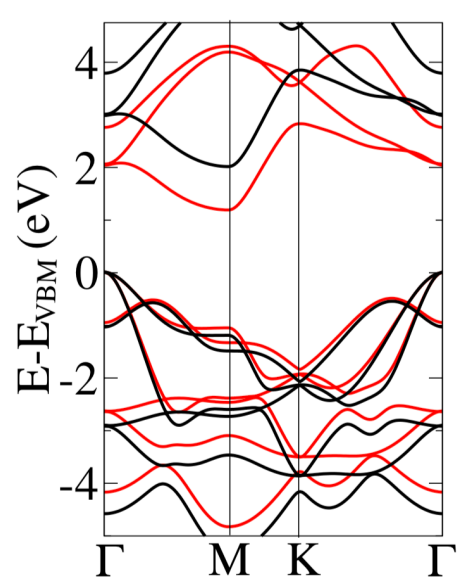

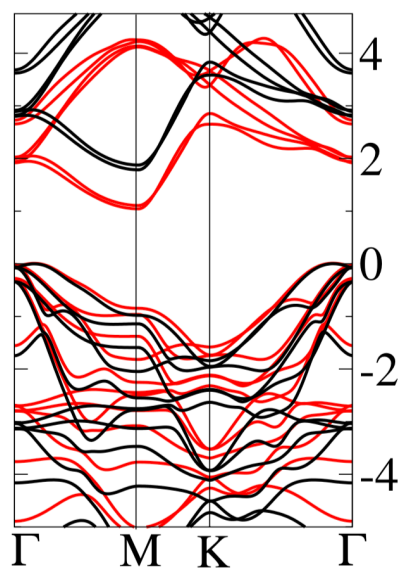

b)

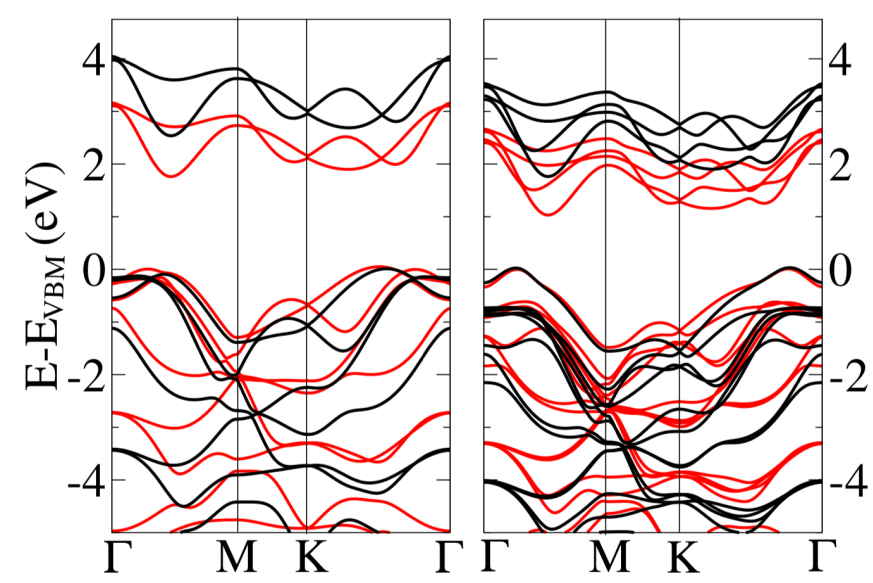

FIG. 3. (Colour online) Bandstructure for (a) $\mathrm{HfS}_{2}$ and (b) $\mathrm{PtS}_{2}$ in their monolayer (left-hand side panels) and bilayer (righthand panels) form. The red and black bands correspond to results obtained with the GGA and HSE functionals, respectively.

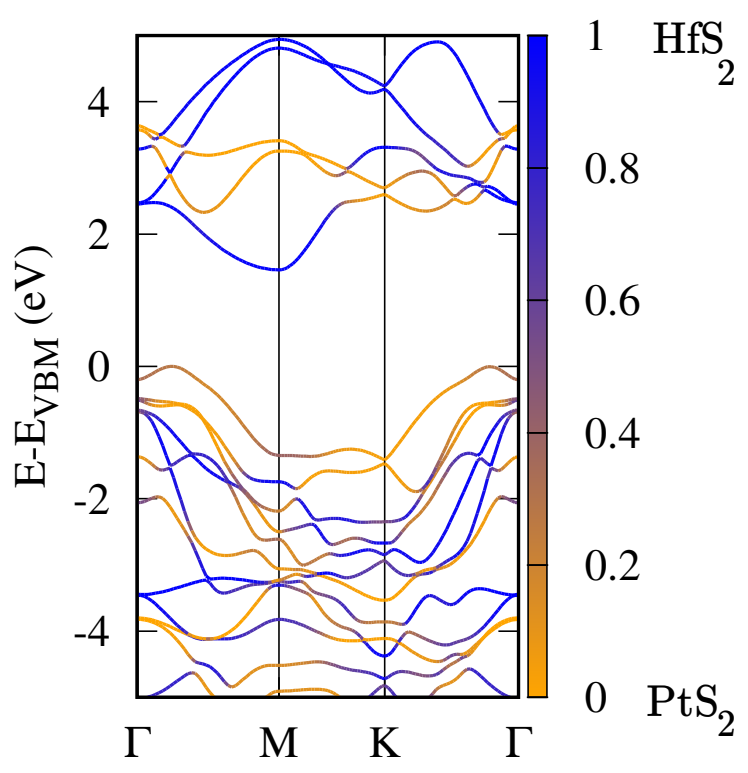

FIG. 4. (Colour online) Bandstructure of the $\mathrm{HfS}_{2} / \mathrm{PtS}_{2} \mathrm{HBL}$ calculated with HSE. The band colour encodes the quantity $f=\mathrm{DOS}_{\mathrm{HfS}_{2}} /\left(\mathrm{DOS}_{\mathrm{HfS}_{2}}+\mathrm{DOS}_{\mathrm{PtS}_{2}}\right)$, namely the projection of a particular eigenstate on the different layers. Blue bands are localised on the $\mathrm{HfS}_{2}$ layer, orange ones on the $\mathrm{PtS}_{2}$ layer.

the individual layers. As such it is useful to attribute to each energy point, $\epsilon_{n \mathbf{k}}$, in the bandstructure a layer character. This is obtained by computing the following quantity, $f=\mathrm{DOS}_{\mathrm{HfS}_{2}} /\left(\mathrm{DOS}_{\mathrm{HfS}_{2}}+\mathrm{DOS}_{\mathrm{PtS}_{2}}\right)$, where $\operatorname{DOS}_{X}$ is the density of states projected on layer $X$ of the eigenfunction, $\left|\psi_{n \mathbf{k}}\right\rangle$. Thus, for $f=1$ the state is entirely localised on the $\mathrm{HfS}_{2}$ monolayer, while for $f=0$ it is entirely localised on the $\mathrm{PtS}_{2}$ layer. Such information is translated into a colour code in Fig. 4. Clearly the $\mathrm{HfS}_{2} / \mathrm{PtS}_{2} \mathrm{HBL}$ presents a type-II band alignment since the CBM is almost completely localised on $\mathrm{HfS}_{2}$, while the entire valence band is mostly on $\mathrm{PtS}_{2}$. It is interesting to observe that, while the conduction band resembles closely that of the $\mathrm{HfS}_{2}$ monolayer, the valence one is much more similar to that of the $\mathrm{PtS}_{2}$ bilayer, with the $k$-degeneracy discussed before. This suggests that the interlayer interaction affects the two constituent materials differently, meaning that the two constituent monolayers have different susceptibility. The resulting heterobilayer is also an indirect semiconductor with an HSE bandgap of $1.41 \mathrm{eV}$ between the $\mathrm{M}$ point $(\mathrm{CBM})$ and a point along the $\Gamma-\mathrm{M}$ line (VBM). This is smaller than the indirect gaps of all the other structures investigated. In contrast, the direct gap is computed with HSE to be $2.22 \mathrm{eV}$, namely it is smaller than the corresponding one for $\mathrm{HfS}_{2}$ BLs, but larger than that of the $\mathrm{PtS}_{2}$ BLs. This means that the three bilayer structures investigated have all a different absorption edge.

\section{B. Dielectric function}

We now proceed to analyse the dielectric function and in particular its imaginary part, which is proportional to the absorption coefficient. In our discussion we separate the in-plane, $\varepsilon_{\|}$, and out-of-plane, $\varepsilon_{\perp}$, components and consider a spectral range comprised between $0.3 \mathrm{eV}$ and $4.3 \mathrm{eV}$, namely within the solar spectrum range as standardised by the American Society for Testing and Materials [31]. Our HSE results are presented in Fig. 5, where panels (a) and (b) show the total dielectric functions for all the structures investigated, while panels (c) and (d) focus on the decomposition of the dielectric function of the $\mathrm{HfS}_{2} / \mathrm{PtS}_{2} \mathrm{HBL}$ into the inter-layer, intra-layer and mixed contributions.

Let us look first at $\varepsilon_{\|}$for the MLs and the homoge- 

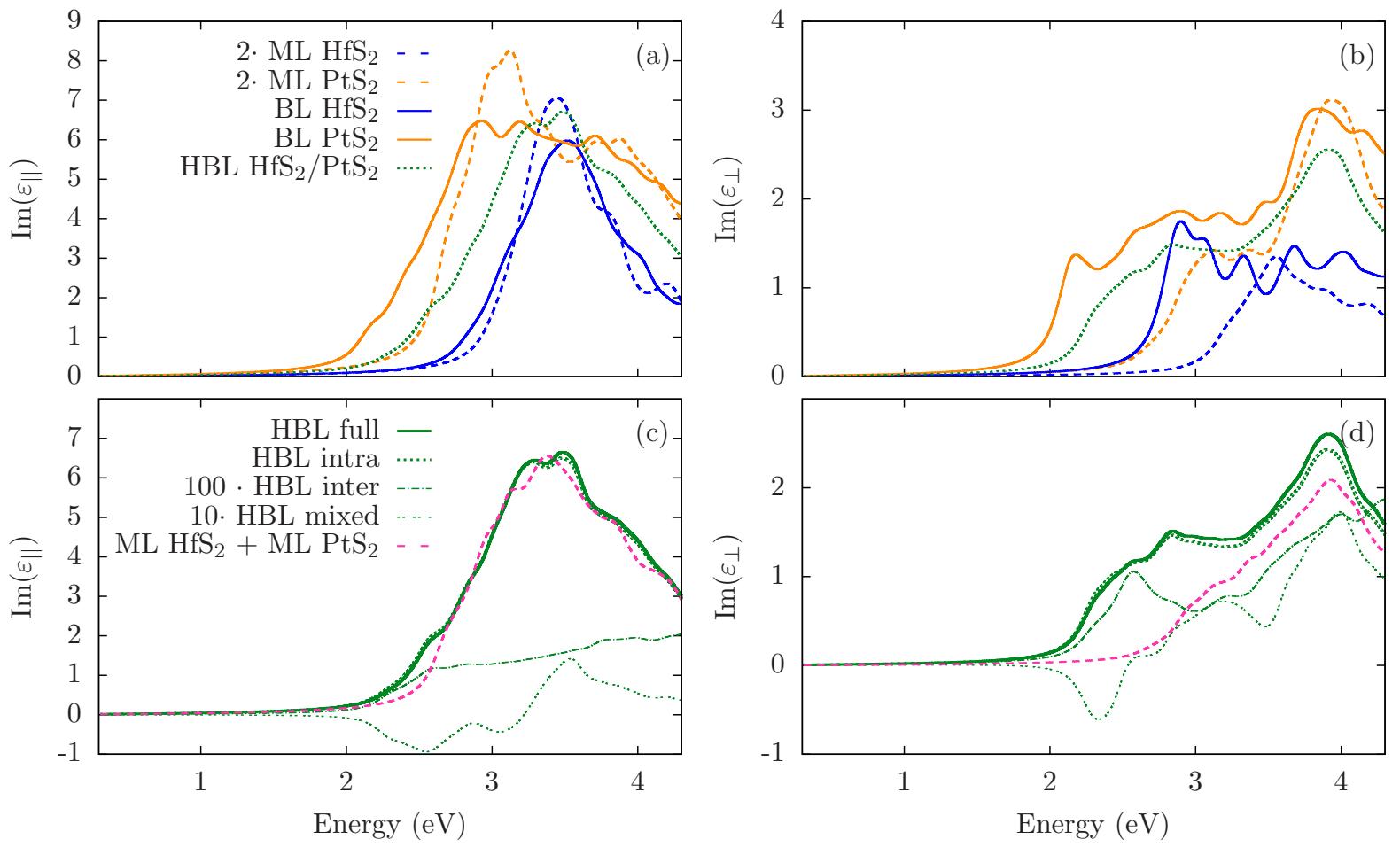

FIG. 5. (Colour online) Imaginary part of the dielectric function for all the structures investigated. (a) in-plane and (b) out-ofplane components of the total dielectric function. Note that in the case of MLs we have scaled $\varepsilon$ by a factor two for comparison. Panels (c) and (d) show the decomposition of the in-plane and out-of-plane dielectric function into intra-layer ("intra"), interlayer ("inter") and "mixed" components for the $\mathrm{HfS}_{2} / \mathrm{PtS} 2 \mathrm{HBL}$ (see Methods section for details). For comparison we also present the sum of the dielectric functions of the $\mathrm{HfS}_{2}$ and $\mathrm{PtS}_{2}$ MLs.

neous BLs [Fig. 5 (a)]. In all cases the absorption edge, as expected, corresponds to the direct HSE bandgap (see Table I). For $\mathrm{HfS}_{2}$ there is little difference in the dielectric function when going from the ML to the BL, despite the change in direct bandgap by about $300 \mathrm{meV}$. In contrast, there is a significant red-shift in the case of $\mathrm{PtS}_{2}$. Interestingly, while for $\mathrm{HfS}_{2}$ the in-plane component of $\operatorname{Im}(\varepsilon)$ of the BL is essentially twice that of the ML (note that in Fig. 5 the ML plots have been rescaled by a factor 2 ), this is not the case for $\mathrm{PtS}_{2}$, where the two functions are rather different. Such feature simply reflects the significant band distortion in $\mathrm{PtS}_{2}$ introduced by the inter-layer interaction and it is in agreement with experimental evidence [29]. The situation for the out-of-plane component, $\varepsilon_{\perp}$, is somewhat different [Fig. 5(b)], mostly because now also $\mathrm{HfS}_{2}$ displays an absorption-edge redshift when going from ML to BL, as expected from the corresponding reduction of the direct bandgap. Overall all perpendicular components of the dielectric function of the MLs are different from those of the corresponding BLs.

The dielectric function of the $\mathrm{HfS}_{2} / \mathrm{PtS}_{2} \mathrm{HBL}$, as expected, is different from all the others. Most importantly its absorption edge is always in between that of the homogeneous $\mathrm{HfS}_{2}$ and $\mathrm{PtS}_{2}$ bilayers, although it is lower than that of all the MLs (in particular for the perpendicular component). This reflects the relative magnitude of the direct gaps of the various structures. A distribution of absorption edges as the one described here, unfortunately, prevents the unique identification of $\mathrm{HfS}_{2} / \mathrm{PtS}_{2}$ HBLs in a mixture containing stacks with different numbers of layers, as those produced by liquid-phase exfoliation [32]. In fact, the most high-throughput means of producing $2 \mathrm{D}$ hetero-structures is by re-aggregating $2 \mathrm{D}$ materials previously exfoliated. In the process, however, one retains little control of the number of layers making the various structures, so that together with heterobilayers one will find in the mixture homo-bilayers, monolayers and a multitude of other structures comprising more than two layers [32-34]. As the lower part of the absorption is always dominated by structures consisting of $\mathrm{PtS}_{2}$, the absorption edge of $\mathrm{HfS}_{2} / \mathrm{PtS}_{2}$ HBLs will never be spectrally separated, hence such structure will not be detectable by a single optical measurement.

The absorption edge of the $\mathrm{HfS}_{2} / \mathrm{PtS}_{2} \mathrm{HBL}$ is just above $2 \mathrm{eV}$, where we find the first direct, inter-layer, transition. However, close to the two VBM there are several intra-layer transitions within $\mathrm{PtS}_{2}$ available. These have an energy just above that of the direct gap (interlayer), so that they can contribute close to the absorption 
edge. In order to identify the different types of transition, in panel (c) and (d) of Fig. 5 we decompose the dielectric function of $\mathrm{HfS}_{2} / \mathrm{PtS}_{2} \mathrm{HBL}$ into its inter-layer, intra-layer and mixed contributions and these are compared with the sum of the dielectric functions of the individual isolated monolayers. Interestingly, while the inplane component $\varepsilon_{\|}$of the HBL is essentially the sum of those of the two monolayers (except for a small shoulder just below $2.5 \mathrm{eV}$ ), the out-of-plane part is significantly different, since its absorption edge is redshifted by about $0.5 \mathrm{eV}$ with respect to the sum of the monolayers. This is a similar behaviour to that found when going from monolayers to homogeneous bilayers.

When looking at the different layer-resolved components the most striking feature concerns their relative amplitude. In fact the spectrum is dominated by the intra-layer component with the inter-layer one accounting for only about $1 \%$ of the total spectrum (note that the inter-layer $\varepsilon$ has been multiplied by a factor 100 in Fig. 5). The mixed component instead contributes to about $10 \%$ and can also take negative values. This essentially means that inter-layer excitons indeed are available but they produce an absorption signal about 100 times smaller than their intra-layer counterparts. This is the same order of magnitude as the factor 200 found in resonant photocurrent measurements of the $\mathrm{MoSe}_{2} / \mathrm{WSe}_{2}$ HBL [35]. It has to be said that the spectra calculated here do not take into account the exciton binding energy. In bilayers both intra- and inter-layer excitons can have binding energies that are some fraction of an eV. However, the latter energy is generally significantly smaller than the former [36-38]. Thus, the actual relative positions of the excitations can be different from those calculated here, with the $\mathrm{PtS}_{2}$ intra-layer excitations being at a lower energy than the inter-layer excitations. The main reason for such a small amplitude of inter-layer transitions has to be associated with the large spatial separation of the electron and hole wave-functions, which reside on different layers and make the dipole matrix elements small.

In order to enhance the amplitude of the interlayer transition, we explore the effect of compressing the stack. This is obtained by incrementally decreasing the interlayer distance of the HBL from the equilibrium value of $d=5.2 \AA$ to $d=4.0 \AA$. The corresponding spectra are depicted in Fig. 6. There are two clear features appearing as the inter-layer distance is reduced, common to both the in-plane and out-of-plane component of the dielectric function. On the one hand, there is a continuous red-shift of the absorption edge, which is sharper for the in-plane component than for that out of plane. This is as large as $1 \mathrm{eV}$ for an inter-layer distance reduction of $1 \AA$. On the other hand, we can observe the formation of a well-isolated absorption peak just at the absorption edge, in particular for small interlayer distances. Thus, we observe that reducing $d$ has profound effects on the dielectric function, which is significantly modified. Such modifications are more pronounced for the out-of-plane component, for which the formation of the new peak at the absorption edge is counterbalanced by a significant increase in amplitude in the spectral region $E>3 \mathrm{eV}$.

These new features of the dielectric function can be understood by looking at the evolution of the bandstructure upon compression, presented in Fig. 7. We observe that, while the conduction bands are little affected by the change in interlayer distance, the valence bands are significantly altered. In particular at small $d$ the top of the valence band splits from the rest of the manifold and already for $d>4.8 \AA$ there is the formation of an impurity band. This contributes to closing the bandgap, which remains indirect but it is reduced by approximately $0.5 \mathrm{eV}$. Interestingly, despite the formation of the impurity band, the dispersion around the VBM is not significantly modified and the VBM degeneracy is preserved. Even more interesting is the fact that the VBM and CBM are localised on the $\mathrm{PtS}_{2}$ and $\mathrm{HfS}_{2}$ layer, respectively, thus the HBL has still a type-II bandstructure. This is in contrast with the rest of the valence bands, which are now formed by hybrid states with almost equal contributions of the two layers.

Thus, we can clearly attribute the peak in the dielectric function at the absorption edge to transitions between the impurity valence band and the lowest conduction band. These are inter-layer in nature, and in fact we observe a significant increase of the inter-layer absorption with compression [see Fig. 6(a) and Fig. 6(e)]. Such features suggest an overall enhanced charge separation, which is promoted both by the presence of layerseparated excitons and by the fact that these form at transitions across an indirect band-gap. For the smallest interlayer distance investigated, $4.0 \AA$, the intra-layer, inter-layer and mixed component of the dielectric function have comparable magnitudes at the absorption edge, while for energies larger than $3 \mathrm{eV}$ the intra-layer component dominates again. This is because in such high energy range the available transitions now involve the lower part of the valence manifold. As a final observation we remark that in all cases the out-of-plane component of the inter-layer dielectric function is significantly larger than the in-plane component.

In order to estimate the pressure needed to yield the layer distances investigated, and hence to monitor the evolution of the inter-layer dielectric function, we compute the pressure, $p$, as a function of the layer distance, $d$. This is simply given by

$$
p=-\frac{\partial E}{\partial V},
$$

where $E$ is the total energy and $V$ is the cell volume, given by the in-plane cell area times the interlayer distance $d$. Our results are plotted in Fig. 8, where we can observe that one needs a pressure of $\simeq 38 \mathrm{GPa}$ to compress the $\mathrm{HfS}_{2} / \mathrm{PtS}_{2} \mathrm{HBL}$ to an interlayer distance of $d=4.4 \AA$. Although this is a rather significant pressure, which exceeds what is possible in standard experimental investigations, it is still lower than the metallisation 

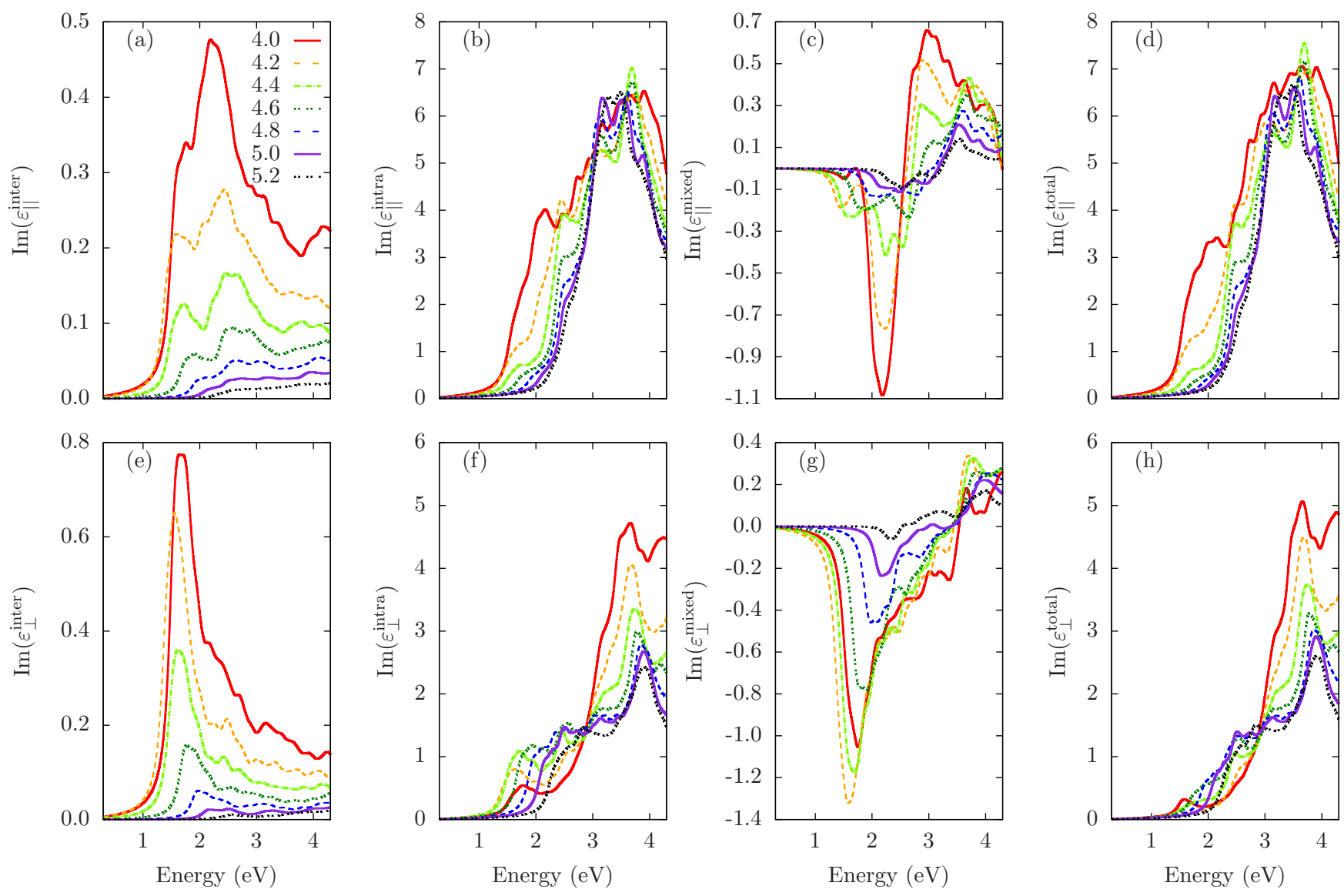

FIG. 6. (Colour online): Imaginary part of the dielectric function as function of the interlayer distance $d$. In panel (a) through (d) we show the inter-layer, intra-layer and mixed parts together with the total spectrum for the in-plane component of $\varepsilon$. Panels (e) through (h) report the same quantities for the out-out-of-plane component.

pressure predicted for other 2D systems, e.g. $68 \mathrm{GPa}$ for $\mathrm{MoS}_{2}$ [39].

\section{SUMMARY AND OUTLOOK}

Our rationale behind vertically stacking TMDs in a LEGO fashion was to explore whether one could find unique and experimentally distinguishable peaks in the dielectric function, which could be unequivocally attributed to inter-layer transitions. Ideally these should be present at energies where no other transitions are available so that they can be uniquely identified, a condition met by heterostructures with type-II band alignment. Furthermore, ideally one wishes to find these features at an energy where other homogeneous structures (multilayers of the same TMD) have no absorption. This situation may enable one to identify inter-layer transitions in heterostructure mixtures produced by liquid-phase processing. Based on these criteria we have used previously published $G W$ data to identify $\mathrm{HfS}_{2} / \mathrm{PtS}_{2}$ hetero-bilayer as a possible candidate.
We have then quantitatively studied the in-plane and out-of-plane components of the dielectric function for $\mathrm{HfS}_{2}$ and $\mathrm{PtS}_{2}$ mono-layers and bilayers, and for a $\mathrm{HfS}_{2} / \mathrm{PtS}_{2}$ hetero-bilayer. In particular we have decomposed the dielectric function into intra-layer, inter-layer and mixed components, so that the specific nature of the transitions can be distinguished. This is obtained by projecting the dipole matrix elements on the relevant layers. We find that the intra-layer component of the dielectric function exceeds the inter-layer component by a factor 100. Furthermore, the mixed component, which does not bare a transparent physical interpretation, is also larger than the inter-layer component and may be negative. Thus, it appears that, although inter-layer transitions are located predominantly at the absorption edge, they cannot be unequivocally separated from the intra-layer transitions. In addition we find that, although the absorption edge of the $\mathrm{HfS}_{2} / \mathrm{PtS}_{2}$ hetero-bilayer is at lower energy than that of the homogeneous $\mathrm{HfS}_{2}$ bilayers, it is at higher energy than that of the $\mathrm{PtS}_{2}$ bilayers. This is because the $\mathrm{PtS}_{2}$ bandstructure is very sensitive to the layer interaction and hence to the number of layers in the 

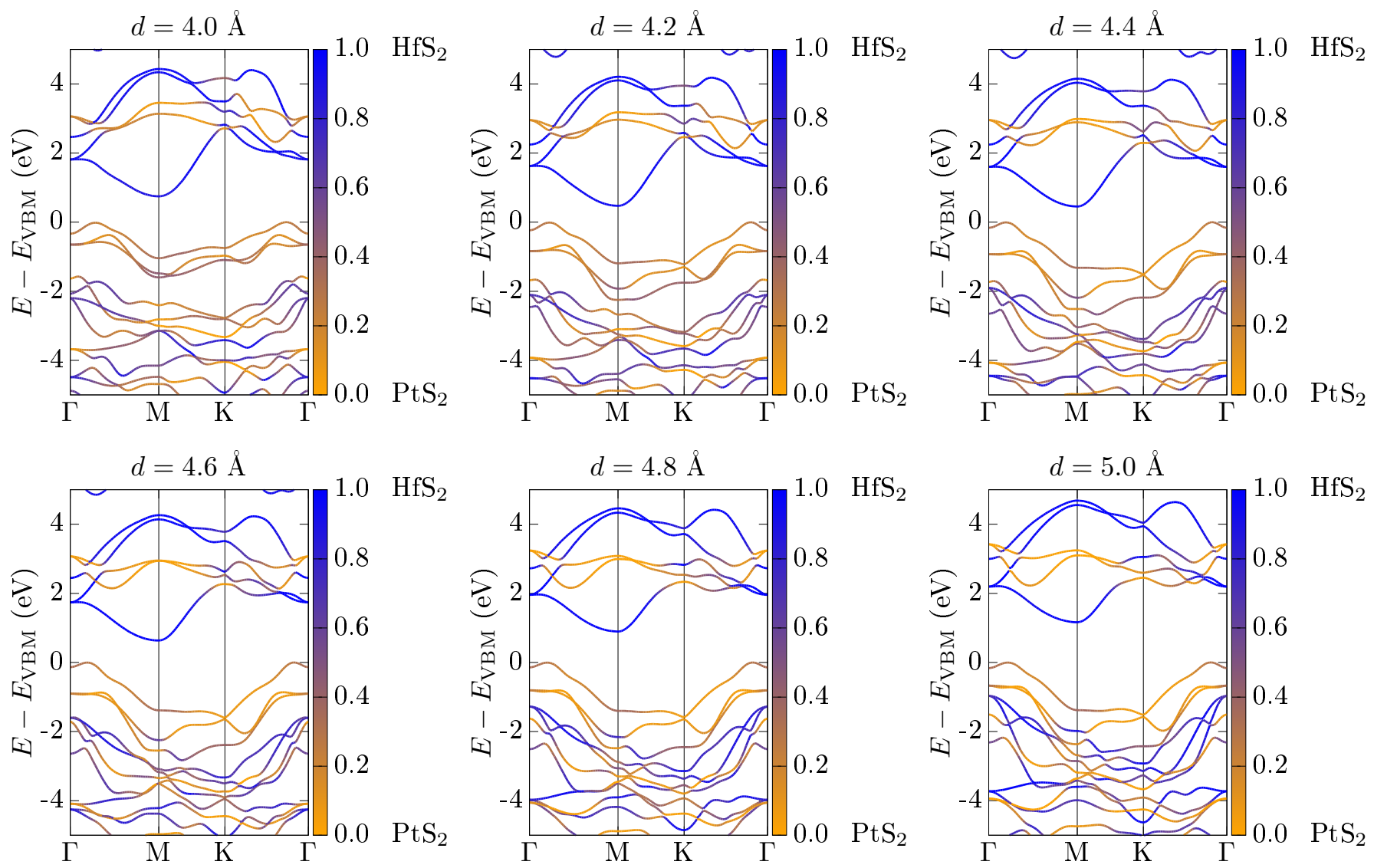

FIG. 7. (Colour online): Bandstructure of the $\mathrm{HfS}_{2} / \mathrm{PtS}_{2} \mathrm{HBL}$ calculated with HSE as a function of the interlayer distance $d$. The band colour encodes the quantity $f=\mathrm{DOS}_{\mathrm{HfS}_{2}} /\left(\mathrm{DOS}_{\mathrm{HfS}_{2}}+\mathrm{DOS}_{\mathrm{PtS}_{2}}\right)$, namely the projection of a particular eigenstate on the different layers. Blue bands are localised on the $\mathrm{HfS}_{2}$ layer, orange ones on $\mathrm{PtS}_{2}$. Note the formation of a split-off band upon compression.

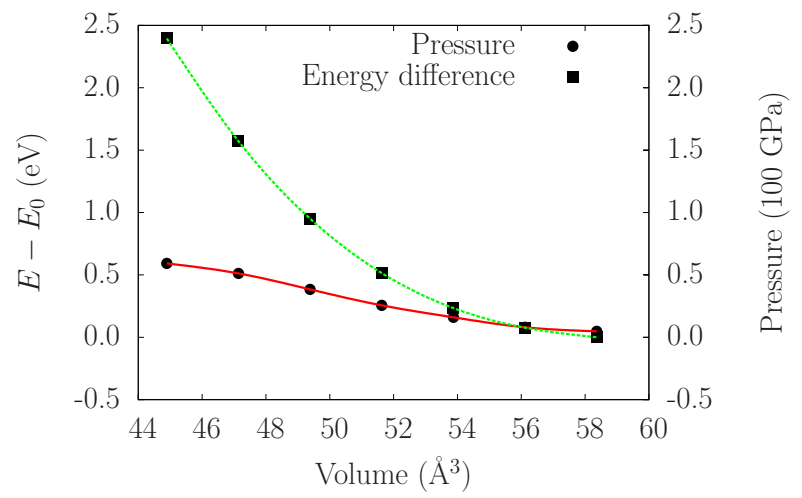

FIG. 8. (Colour online): Energy curve as a function of volume for each interlayer distance $d$ (squares) and its derivative corresponding to the pressure (circles). The energy was shifted by $E_{0}$, the total energy at the equilibrium distance $d=5.2 \AA$. The lines (splines) are a guide for the eye. stack, as reported before [29].

Since the small inter-layer transition amplitude originates from the large spatial separation between the electron and its hole (residing on different layers), we have investigated the changes in the dielectric function as the layer distance is reduced. Indeed, this promotes an increase in the inter-layer dielectric function, together with a significant red-shift of the absorption edge, mostly driven by the formation of a well separated peak. By following the evolution of the bandstructure upon decreasing the interlayer distance we can attribute such behaviour to the formation of a valence impurity band, well separated from the rest of the valence manifold. Most interestingly, the type-II band alignment is maintained even under severe compression, so that the split-off peak at the absorption edge is mostly composed of inter-layer transitions.

In conclusion, we have shown that TMD heterostructures under severe compression present bandstructures and dielectric properties different from those of the 
constituent layers and different from those expected from simple electrostatic models. Here we have discussed one example in which the type-II band alignment can be preserved under pressure and where pressure can be used to uniquely isolate the inter-layer excitations from the rest of the spectrum.

\section{ACKNOWLEDGMENTS}

We acknowledge financial support by the Qatar National Research Fund through the NPRP 8-090-2-047 CRAQSolar project. All calculations were performed on clusters maintained by the Trinity Centre for High Performance Computing. These clusters were funded through grants from Science Foundation Ireland and the Irish Higher Education Authority, through its PRTLI program.
[1] M. Bernardi, M. Palummo, and J. C. Grossman, Extraordinary sunlight absorption and one nanometer thick photovoltaics using two-dimensional monolayer materials, noop Nano Lett. 13, 3664 (2013).

[2] Y. Sun, R. Wang, and K. Liu, Substrate induced changes in atomically thin 2-dimensional semiconductors: Fundamentals, engineering, and applications, noop Appl. Phys. Rev. 4, 011301 (2017).

[3] W. Choi, N. Choudhary, G. H. Han, J. Park, D. Akinwande, and Y. H. Lee, Recent development of twodimensional transition metal dichalcogenides and their applications, noop Mater. Today 20, 116 (2017).

[4] P. Ajayan, P. Kim, and K. Banerjee, Two-dimensional van der waals materials, noop Phys. Today 69, 38 (2016).

[5] A. K. Geim and I. V. Grigorieva, Van der Waals heterostructures, noop Nature 499, 419 (2013).

[6] N. Choudhary, J. Park, J. Y. Hwang, H.-S. Chung, K. H. Dumas, S. I. Khondaker, W. Choi, and Y. Jung, Centimeter scale patterned growth of vertically stacked few layer only $2 \mathrm{D} \mathrm{MoS}_{2} / \mathrm{WS}_{2}$ van der Waals heterostructure, noop Sci. Rep. 6, 25456 (2016).

[7] P. Rivera, H. Yu, K. L. Seyler, N. P. Wilson, W. Yao, and $\mathrm{X}$. Xu, Interlayer valley excitons in heterobilayers of transition metal dichalcogenides, noop Nature Nanotechnol. 13, 1004 (2018).

[8] J. Yan, C. Ma, Y. Huang, and G. Yang, Tunable Control of Interlayer Excitons in $\mathrm{WS}_{2} / \mathrm{MoS}_{2}$ Heterostructures via Strong Coupling with Enhanced Mie Resonances, noop Adv. Sci. 6, 1802092 (2019).

[9] H. Fang, C. Battaglia, C. Carraro, S. Nemsak, B. Ozdol, J. S. Kang, H. A. Bechtel, S. B. Desai, F. Kronast, A. A. Unal, G. Conti, C. Conlon, G. K. Palsson, M. C. Martin, A. M. Minor, C. S. Fadley, E. Yablonovitch, R. Maboudian, and A. Javey, Strong interlayer coupling in van der Waals heterostructures built from single-layer chalcogenides, noop P. Natl. Acad. Sci. 111, 6198 (2014).

[10] H. Heo, J. H. Sung, S. Cha, B.-G. Jang, J.-Y. Kim, G. Jin, D. Lee, J.-H. Ahn, M.-J. Lee, J. H. Shim, et al., Interlayer orientation-dependent light absorption and emission in monolayer semiconductor stacks, noop Nature Commun. 6, 7372 (2015).

[11] X. Zhu, N. R. Monahan, Z. Gong, H. Zhu, K. W. Williams, and C. A. Nelson, Charge transfer excitons at van der Waals interfaces, noop J. Am. Chem. Soc. 137, 8313 (2015).

[12] Y. Yu, S. Hu, L. Su, L. Huang, Y. Liu, Z. Jin, A. A. Purezky, D. B. Geohegan, K. W. Kim, Y. Zhang, and L. Cao, Equally efficient interlayer exciton relaxation and improved absorption in epitaxial and nonepitaxial $\mathrm{MoS}_{2} / \mathrm{WS}_{2}$ heterostructures, noop Nano Lett. 15, 486 (2014).

[13] K. Andersen, S. Latini, and K. S. Thygesen, Dielectric genome of van der Waals heterostructures, noop Nano Lett. 15, 4616 (2015).

[14] H.-P. Komsa and A. V. Krasheninnikov, Electronic structures and optical properties of realistic transition metal dichalcogenide heterostructures from first principles, noop Phys. Rev. B 88, 085318 (2013).

[15] F. A. Rasmussen and K. S. Thygesen, Computational 2D materials database: electronic structure of transitionmetal dichalcogenides and oxides, noop J. Phys. Chem. C 119, 13169 (2015).

[16] W. Kohn, A. D. Becke, and R. G. Parr, Density functional theory of electronic structure, noop J. Phys. Chem. 100, 12974 (1996).

[17] R. G. Parr and Y. Weitao, noop Density-Functional Theory of Atoms and Molecules (Oxford University Press, 1994).

[18] V. Blum, R. Gehrke, F. Hanke, P. Havu, V. Havu, X. Ren, K. Reuter, and M. Scheffler, Ab initio molecular simulations with numeric atom-centered orbitals, noop Comp. Phys. Comm. 180, 2175 (2009).

[19] V. Havu, V. Blum, P. Havu, and M. Scheffler, Efficient $\mathrm{O}(\mathrm{N})$ integration for all-electron electronic structure calculation using numeric basis functions, noop J. Comp. Phys. 228, 8367 (2009).

[20] X. Ren, P. Rinke, V. Blum, J. Wieferink, A. Tkatchenko, A. Sanfilippo, K. Reuter, and M. Scheffler, Resolution-ofidentity approach to hartree-fock, hybrid density functionals, RPA, MP2 and GW with numeric atom-centered orbital basis functions, noop New J. Phys. 14, 053020 (2012).

[21] J. P. Perdew, K. Burke, and M. Ernzerhof, Generalized gradient approximation made simple, noop Phys. Rev. Lett. 77, 3865 (1996).

[22] A. Tkatchenko and M. Scheffler, Accurate molecular van der Waals interactions from ground-state electron density and free-atom reference data, noop Phys. Rev. Lett. 102, 073005 (2009).

[23] J. Heyd, G. E. Scuseria, and M. Ernzerhof, Hybrid functionals based on a screened coulomb potential, noop J. Chem. Phys. 118, 8207 (2003).

[24] G. Kresse and J. Furthmüller, Efficient iterative schemes for ab initio total-energy calculations using a plane-wave basis set, noop Physical Review B 54, 11169 (1996).

[25] C. Ambrosch-Draxl and J. O. Sofo, Linear optical prop- 
erties of solids within the full-potential linearized augmented planewave method, noop Comp. Phys. Comm. 175, 1 (2006).

[26] R. S. Mulliken, Electronic population analysis on LCAOMO molecular wave functions. I, noop J. Phys. Chem. 23, 1833 (1955).

[27] M. Traving, T. Seydel, L. Kipp, M. Skibowski, F. Starrost, E. E. Krasovskii, A. Perlov, and W. Schattke, Combined photoemission and inverse photoemission study of $\mathrm{HfS}_{2}$, noop Phys. Rev. B 63, 035107 (2001).

[28] C. Kreis, S. Werth, R. Adelung, L. Kipp, M. Skibowski, E. E. Krasovskii, and W. Schattke, Valence and conduction band states of $\mathrm{HfS}_{2}$ : From bulk to a single layer, noop Phys. Rev. B 68, 235331 (2003).

[29] Y. Zhao, J. Qiao, P. Yu, Z. Hu, Z. Lin, S. P. Lau, Z. Liu, W. Ji, and Y. Chai, Extraordinarily strong interlayer interaction in 2D layered $\mathrm{PtS}_{2}$, noop Adv. Mater. 28, 2399 (2016).

[30] R. A. B. Villaos, C. P. Crisostomo, Z.-Q. Huang, S.-M. Huang, A. A. B. Padama, M. A. Albao, H. Lin, and F.-C. Chuang, Thickness dependent electronic properties of $\mathrm{Pt}$ dichalcogenides, noop npj 2D Mater. App. 3, 2 (2019).

[31] Standard tables for reference solar spectral irradiances: direct normal and hemispherical on $37^{\circ}$ tilted surface, noop ASTM G173 - $03 \mathbf{1 4 . 0 4}$ (2012).

[32] S. Witomska, T. Leydecker, A. Ciesielski, and P. Samorì, Production and patterning of liquid phase-exfoliated $2 \mathrm{~d}$ sheets for applications in optoelectronics, noop Advanced Functional Materials 29, 1901126 (2019).

[33] H. Wang, F. Liu, W. Fu, Z. Fang, W. Zhou, and Z. Liu, Two-dimensional heterostructures: fabrication, charac- terization, and application, noop Nanoscale 6, 12250 (2014).

[34] X. Zhang, H. Nan, S. Xiao, X. Wan, X. Gu, A. Du, Z. Ni, and K. K. Ostrikov, Transition metal dichalcogenides bilayer single crystals by reverse-flow chemical vapor epitaxy, noop Nature communications 10, 598 (2019).

[35] J. S. Ross, P. Rivera, J. Schaibley, E. Lee-Wong, H. Yu, T. Taniguchi, K. Watanabe, J. Yan, D. Mandrus, D. Cobden, W. Yao, and X. Xu, Interlayer exciton optoelectronics in a $2 \mathrm{~d}$ heterostructure pn junction, Nano Letters 17, 638 (2017), pMID: 28006106, https://doi.org/10.1021/acs.nanolett.6b03398.

[36] S. Ovesen, S. Brem, C. Linderälv, M. Kuisma, T. Korn, P. Erhart, M. Selig, and E. Malic, Interlayer exciton dynamics in van der waals heterostructures, noop Communications Physics 2, 23 (2019).

[37] S. Latini, K. T. Winther, T. Olsen, and K. S. Thygesen, Interlayer excitons and band alignment in mos $2 / \mathrm{hbn} /$ wse2 van der waals heterostructures, noop Nano letters 17, 938 (2017).

[38] E. Torun, H.P.C. Miranda, A. Molina-Sánchez, and L. Wirtz, Interlayer and intralayer excitons in mos 2 /ws 2 and mose 2 /wse 2 heterobilayers, noop Physical Review B 97, 245427 (2018).

[39] A. P. Nayak, T. Pandey, D. Voiry, J. Liu, S. T. Moran, A. Sharma, C. Tan, C.-H. Chen, L.-J. Li, M. Chhowalla, J.-F. Lin, A. K. Singh, and D. Akinwande, Pressuredependent optical and vibrational properties of monolayer molybdenum disulfide, noop Nano Lett. 15, 346 (2014). 